Supporting Information For

\title{
Incorporation of a Basil-Seed-Based Surface Enhanced Raman Scattering Sensor with a Pipet for Detection of Melamine
}

Ningning Zhou, ${ }^{\dagger},{ }^{\ddagger}$ Qitao Zhou, ${ }^{\dagger}$ Guowen Meng, ${ }^{*}{ }^{\dagger}$ Zhulin Huang, ${ }^{\dagger}$ Yan Ke ${ }^{\dagger},+$ Jing Liu ${ }^{\dagger}$ and Nianqiang $\mathrm{Wu}^{*}, \S$

${ }^{\dagger}$ Key Laboratory of Materials Physics, CAS Center for Excellence in Nanoscience, and Anhui Key Laboratory of Nanomaterials and Nanotechnology, Institute of Solid State Physics, Chinese Academy of Sciences, Hefei 230031 (P. R. China)

$\$$ University of Science and Technology of China, Hefei 230026 (P. R. China)

${ }^{\S}$ Department of Mechanical and Aerospace Engineering, West Virginia University, P. O. Box 6106, Morgantown, West Virginia 26506, United States

* E-mail: gwmeng@issp.ac.cn

* E-mail: Nick.Wu@ mail.wvu.edu 


\section{Table of Contents}

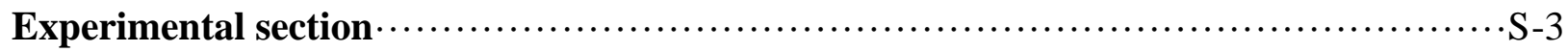

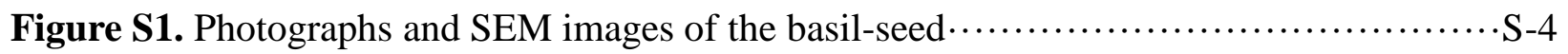

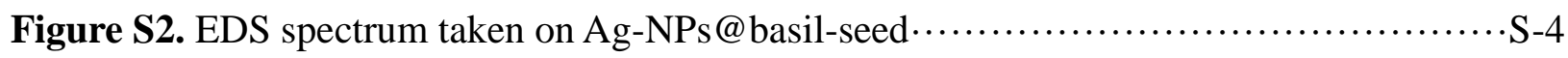

Figure S3. UV-Visible spectra of the basil-seeds before and after deposition of Ag-NPs $\cdots . . .$. S-5

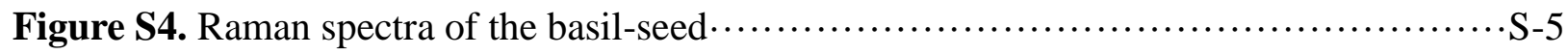

Figure S5. SERS spectra of R6G collected on random spots of the Ag-NPs@ @asil-seed.......S-6

Figure S6. Intensity distribution of SERS spectra of R6G collected on 25 randomly selected Ag-

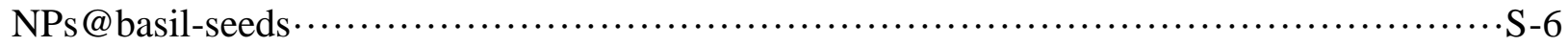

Figure S7. SERS spectra of R6G with different concentrations collected on Ag-NPs@basil-

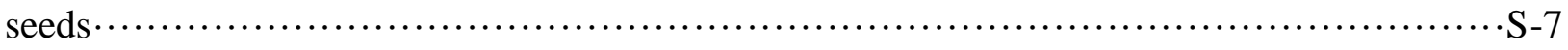

Figure S8. SERS spectra evolution of R6G and average spectra of the same analyte measured in pipet-basil-seed-based SERS device and an open Ag-NPs@ basil-seed substrate ............S-7

Figure S9. SERS spectra of methyl parathion obtained at two excitation wavelengths $\cdots \cdots \cdots \cdot$...S 8

Figure S10. UV-vis spectra of methyl parathion and pure orange juice $\ldots \ldots \ldots \ldots \ldots \ldots \ldots \ldots, 8$

Figure S11. SERS spectra of melamine by using the Ag-NPs@basil-seed soaked in milk in the

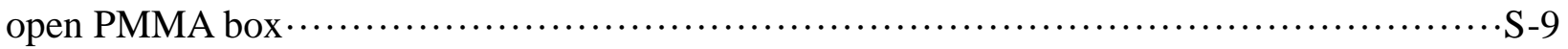




\section{Experimental Section}

Materials. Silver nitrate $\left(\mathrm{AgNO}_{3}\right)$, ammonium hydroxide $\left(\mathrm{NH}_{3} \cdot \mathrm{H}_{2} \mathrm{O}\right)$ and glucose were obtained from Sinopharm Chemical Reagent limited corporation. Rhodamine 6G (R6G) was purchased from Sigma Aldrich. Methyl parathion was purchased from Aladdin. Melamine was obtained from J\&K Chemicals Ltd. Milli-Q deionized (DI) water (resistivity $=18.2 \mathrm{M} \Omega \bullet \mathrm{cm}$ ) was used for all experiments.

Deposition of Ag-NPs onto the network of the basil-seed. Ag-NPs were loaded onto the network of the basil-seed via Ag mirror reaction. Briefly, diluted $10 \% \mathrm{NH}_{3} \cdot \mathrm{H}_{2} \mathrm{O}$ was injected into $100 \mathrm{~mL}$ of $25 \mathrm{mM}$ aqueous $\mathrm{AgNO}_{3}$ under stirring until yellow precipitate disappeared. The basil-seeds were soaked in the silver ammonia solution and kept for $20 \mathrm{~min}$. Then the basil-seeds were filtered to remove the excessive silver ammonia solution. Subsequently, $100 \mathrm{~mL}$ of $50 \mathrm{mM}$ glucose solution was added under stirring and heated at $50{ }^{\circ} \mathrm{C}$. After 20 minutes, the basil-seeds turned gray, and the obtained Ag-NPs@ basil-seeds were cleaned with DI water several times and dried in a drying oven.

Device Preparation. The PMMA box $\left(29 \times 22 \times 10 \mathrm{~mm}^{3}\right)$ had a cavity with an inner diameter of $6 \mathrm{~mm}$ and a height of $3 \mathrm{~mm}$. Two inner channels of $5 \mathrm{~mm}$ in a diameter and $10 \mathrm{~mm}$ in length were opened to connect the cavity in the PMMA box. A regular transfer pipet with $1 \mathrm{~mL}$ of volume was cut into two segments, which were then inserted into the two channels on the two opposite sides of the PMMA box, respectively. Next, a dried Ag-NPs@basil-seed was put into the cavity of the PMMA box. The top surface PMMA box is covered by the glass slide, and sealed with a thawing candle.

SERS Measurement. Orange juice spiked with different concentrations of methyl parathion was sucked into the pipet-Ag-NPs@basil-seed device to wet the Ag-NPs@ basil-seed and kept for 10 min. The SERS spectra were acquired from the Ag-NPs@basil-seed in the pipet-AgNPs@basil-seed device with a confocal microprobe Raman system (Renishaw, InVia). The Raman signals were recorded using a $633 \mathrm{~nm}$ laser excitation with a $50 \times$ objective at $0.85 \mathrm{~mW}$ of power. The integral time was $5 \mathrm{~s}$.

Melamine in milk was measured with the pipet-Ag-NPs@basil-seed device as shown in Figure 1. The analyte solution was sucked to fill the PMMA box by simply employing a pressure on the transfer pipet. The Ag-NPs@basil-seed substrate can directly contact with the analyte solution and enrich the target molecules. After incubation for several minutes, the analyte molecules were fully adsorbed on the Ag-NPs@basil-seed. Afterwards, the laser beam was focused on the Ag-NPs@ basil-seed to collect the SERS signal.

Characterization. The morphology and microstructures of samples were characterized by a field-emission scanning electron microscope (SEM, SU8020) and a transmission electron microscope (TEM, JEM-2010.). For TEM characterization, a strip of minced resultant products was put on a $\mathrm{Cu}$ grid. The chemical composition of the samples was recorded with an energydispersive X-ray spectroscopy (EDS, Oxford). 

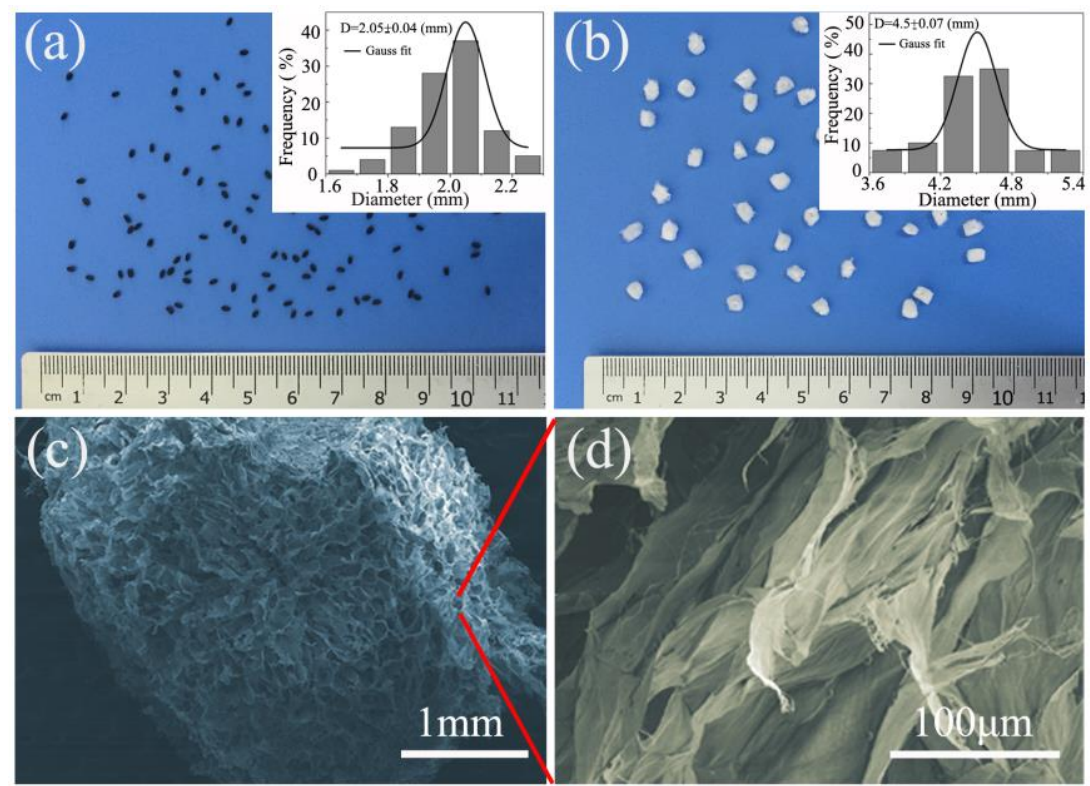

Figure S1. (a) Optical photograph of the dry basil-seeds. The inset is the corresponding size distribution of dry basil-seeds. (b) Optical photograph of the soaked basil-seeds after freezedrying. The inset is the corresponding size distribution of the soaked basil-seeds after freezedrying. (c) and (d) SEM images of the soaked basil-seeds after freeze-drying.

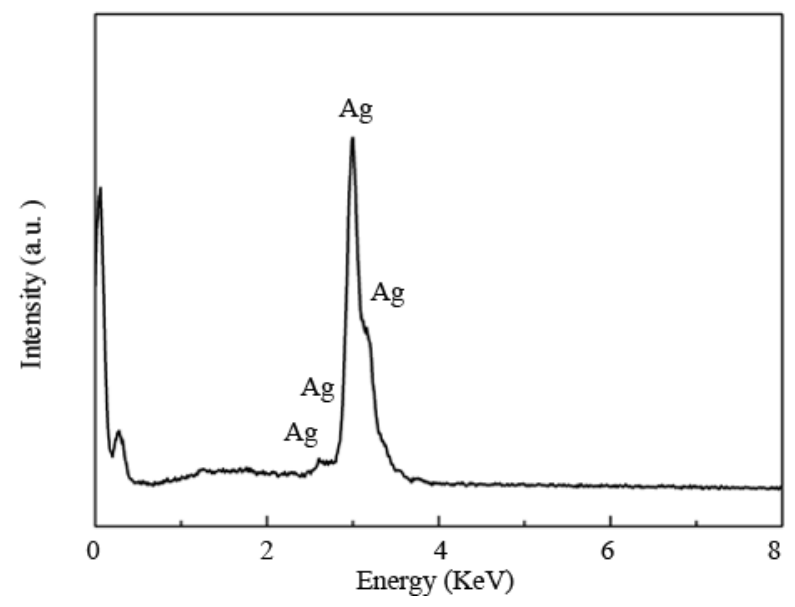

Figure S2. EDS spectrum taken from the Ag-NPs deposited on the surface of the basil-seed in Figure 2f. 


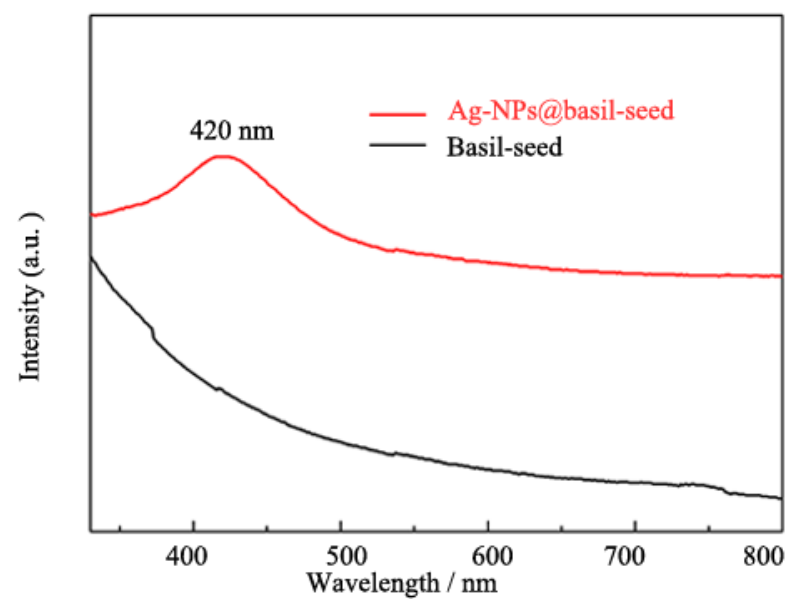

Figure S3. UV-Visible spectra of the basil-seeds before and after deposited with Ag-NPs.

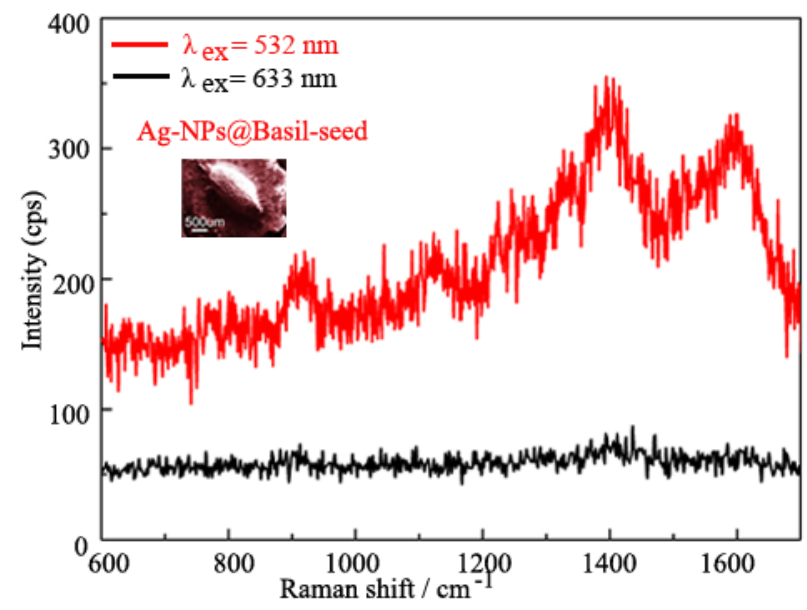

Figure S4. Raman spectra of basil-seed used as scaffold for loading SERS-active metal NPs, obtained under the excitation of $532 \mathrm{~nm}$ and $633 \mathrm{~nm}$ lasers, respectively. 


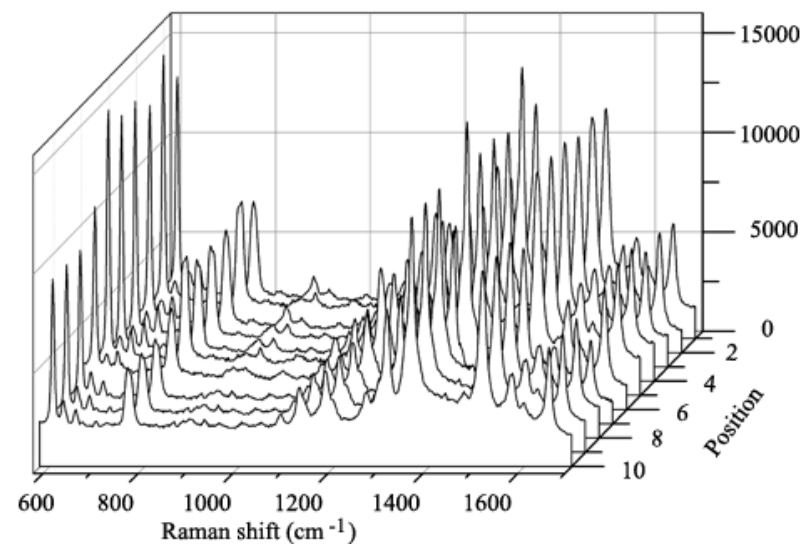

Figure S5. SERS spectra of $1 \mu \mathrm{M}$ R6G collected on 10 randomly selected spots from the AgNPs@basil-seed under the excitation of the 633 nm laser.

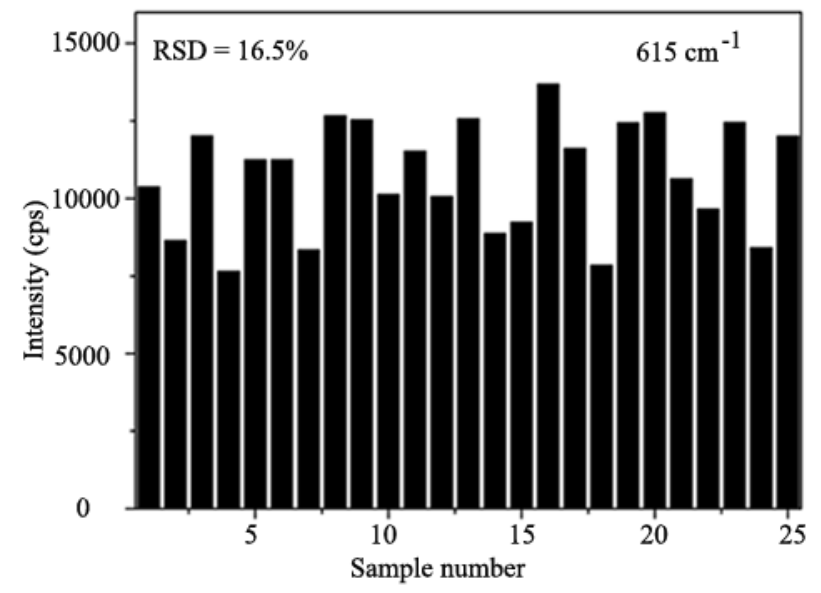

Figure S6. Intensity distribution of SERS spectra of $1 \mu \mathrm{M} \mathrm{R} 6 \mathrm{G}$ at $615 \mathrm{~cm}^{-1}$ collected on 25 randomly selected Ag-NPs@ @asil-seed sensors under the excitation of the $633 \mathrm{~nm}$ laser. 


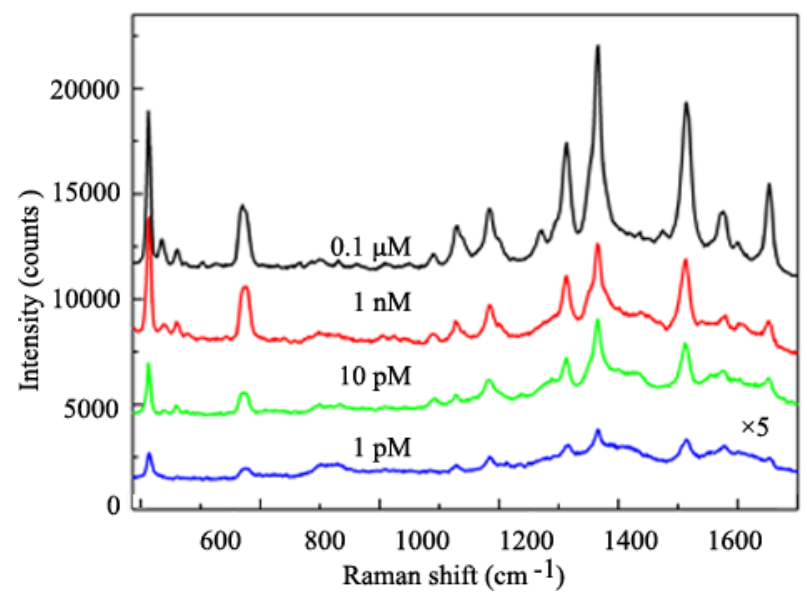

Figure S7. SERS spectra of R6G at different concentrations collected on the Ag-NPs@basilseeds under the excitation of the $633 \mathrm{~nm}$ laser.
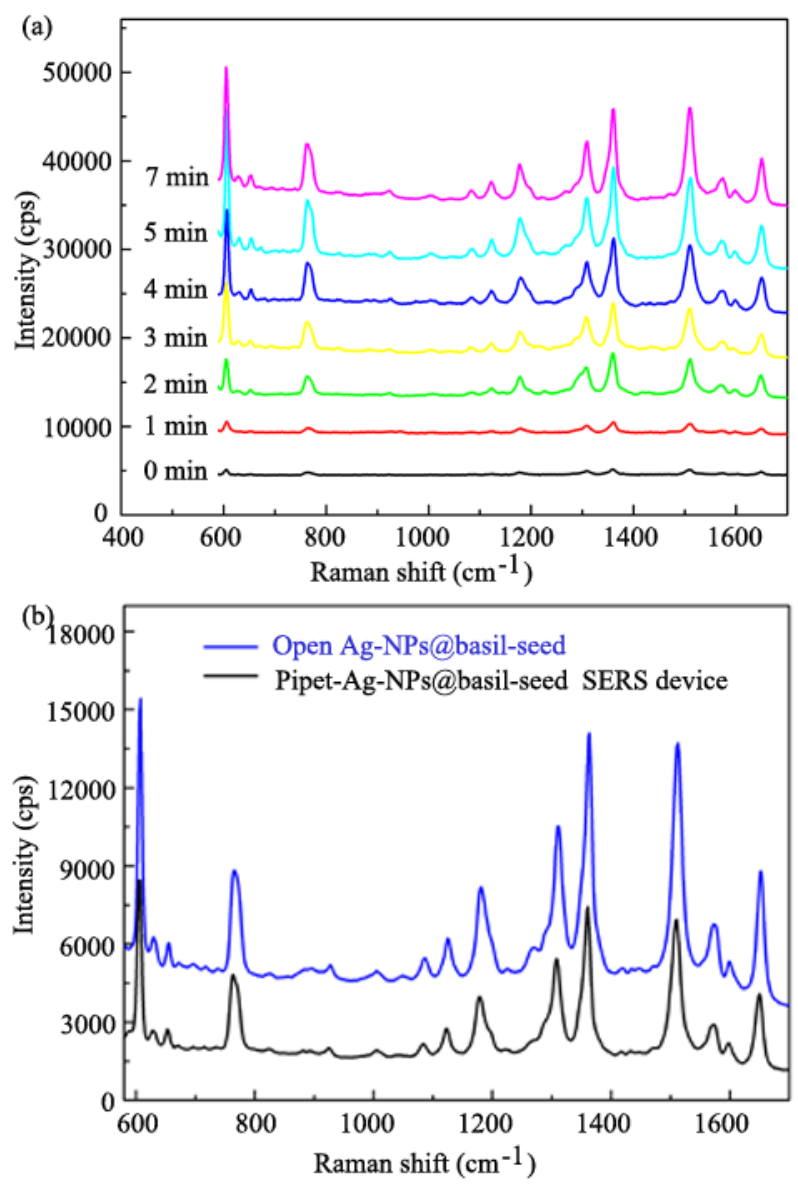

Figure S8. (a) SERS spectra evolution of R6G $(1 \mu \mathrm{M})$ using pipet-Ag-NPs@ basil-seed SERS device at intervals of 1 min under the excitation of the $633 \mathrm{~nm}$ laser. (b) Average spectra of the same analyte measured in the pipet-Ag-NPs@basil-seed SERS device and an open AgNPs@basil-seed substrate under the excitation of the $633 \mathrm{~nm}$ laser. 


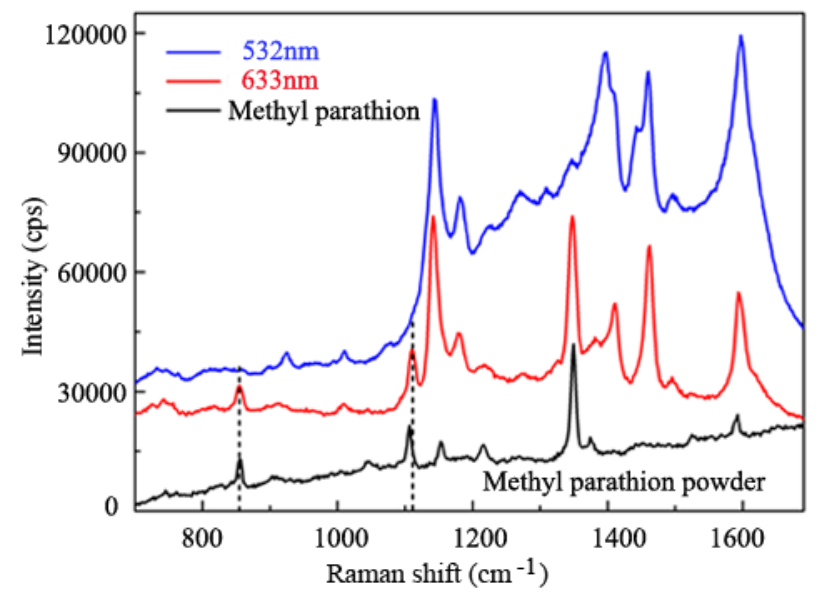

Figure S9. SERS spectra of $(50 \mu \mathrm{M})$ methyl parathion collected from the pipet-Ag-NPs@basilseed SERS device under excitation of the $532 \mathrm{~nm}$ and $633 \mathrm{~nm}$ lasers. The black line was the Raman spectrum of methyl parathion powder.
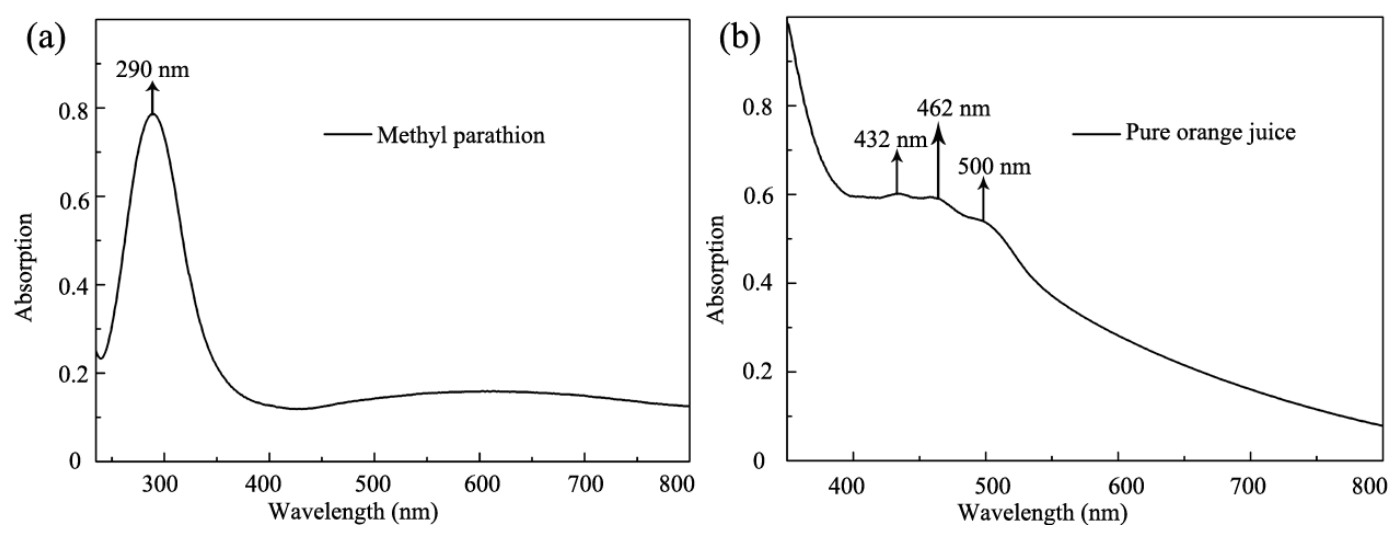

Figure S10. UV-vis spectra of (a) methyl parathion and (b) pure orange juice. 


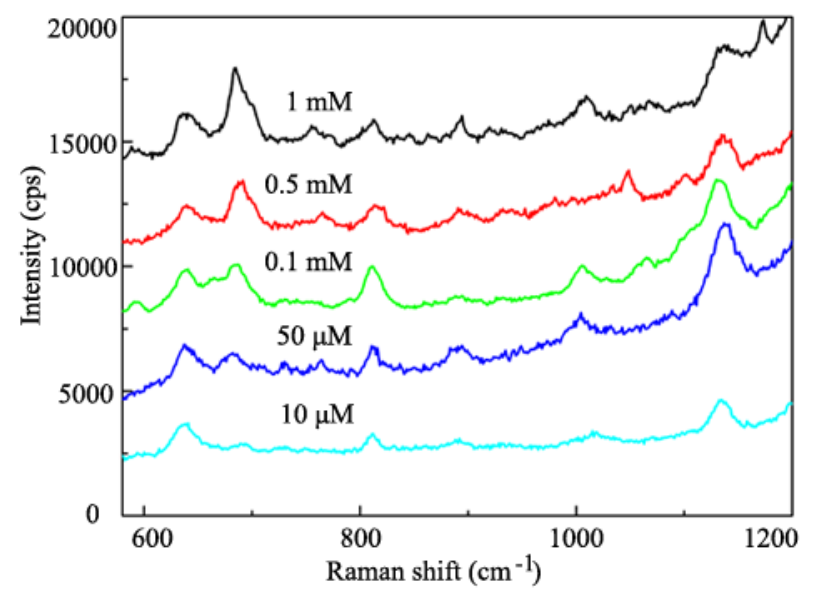

Figure S11. SERS spectra of melamine in milk under the excitation of the 532nm laser using AgNPs@basil-seed soaked in the milk in the open PMMA box. 